\title{
Pulmonary Tuberculosis With Saddle Pulmonary Embolism and Deep Vein Thrombosis: A Rare Case Report
}

\author{
Eihab A. Subahi ${ }^{1}$, Mouhammad J. Alawad ${ }^{1}$, Elabbass A. Abdelmahmuod ${ }^{1}$, Dalal Sibira ${ }^{2}$, Ijaz Kamal ${ }^{1}$ \\ 1. Internal Medicine Department, Hamad Medical Corporation, Doha, QAT 2. Radiology Department, Hamad Medical \\ Corporation, Doha, QAT
}

Corresponding author: Eihab A. Subahi, ehabsubahi@yahoo.com

\begin{abstract}
Pulmonary tuberculosis is a common endemic disease in developing countries but its thrombogenic tendency is not well-studied and established yet. Pulmonary embolism is rarely reported in Mycobacterium tuberculosis infection. There are reports stating the relation of pulmonary embolism (PE) and deep vein thrombosis (DVT) with a severe infection of tuberculosis but no data is available to establish a mutual association between pulmonary tuberculosis and pulmonary thromboembolism. Herein, we report the case of a 51-year-old male who presented with a one-month history of productive cough, shortness of breath, and fever associated with chills and night sweating. He reported an $8 \mathrm{~kg}$ weight loss in the last month. He was found to have pulmonary tuberculosis. On further investigations for leg swelling and tachycardia. Deep vein thrombosis and sub-massive saddle bilateral pulmonary embolism were diagnosed, which was treated with thrombolysis therapy (alteplase). He responded well to initial therapy and was discharged on anticoagulation with anti-tuberculous therapy (ATT).
\end{abstract}

Review began 06/17/2021 Review ended 06/20/2021 Published 06/27/2021

(c) Copyright 2021 Subahi et al. This is an open access article distributed under the terms of the Creative Commons Attribution License CC-BY 4.0., which permits unrestricted use, distribution, and reproduction in any medium, provided the original author and source are credited.
Categories: Internal Medicine, Infectious Disease

Keywords: pulmonary tuberculosis, pulmonary embolism, deep vein thrombosis, anti-tubercular therapy, anticoagulation

\section{Introduction}

Tuberculosis (TB) is defined as an infectious disease caused by Mycobacterium tuberculosis [1]. TB can cause various symptoms and signs depending on the infected organs [1]. The lungs are involved in approximately $90 \%$ of cases while other systems can also be involved like the gastrointestinal tract, genitourinary tract, lymph nodes, bone, muscle, and central nervous system, and this is called extrapulmonary tuberculosis. In addition, it is well-known that a hypercoagulable state associated with tuberculosis may provoke thromboembolism, and this complication occurs in 0.6-1.0 of patients with tuberculosis [1-2]. Although venous thromboembolism (VTE) is considered a rare incident, it should be taken into consideration especially in those with severe pulmonary or disseminated tuberculosis, as there is some evidence that the risk of developing VTE increasing with the severity of the disease [3-4]. Another challenge is the drug interactions particularly between rifampicin, which is the mainstay of treatment, and warfarin or oral anticoagulants [5]. However, to the best of our knowledge, few cases have been reported in the literature about venous thromboembolism in patients with tuberculosis [1,3-4,6]. Therefore, physicians should have a high index of suspicion and PE should be suspected as one of the differential diagnoses in patients with pulmonary TB (PTB) who have hypoxia, tachycardia, sudden onset of chest pain, or increasing shortness of breath. As VTE is associated with high mortality and morbidity [4], therefore, it is important to perform an early diagnosis and initiate immediate and proper treatment [3].

\section{Case Presentation}

We present a case of a 51-year-old Bangladeshi male with a past medical history of uncontrolled diabetes mellites (DM) (last glycated hemoglobin (HBA1c) 13.4\%) who was on oral hypoglycemic medication (metformin/sitagliptin). He came to the emergency department with a one-month history of productive cough, shortness of breath, which was associated with fever and night sweating. He reported an $8 \mathrm{~kg}$ weight loss in the last month. He denied any other complaints like hemoptysis. He works as a private house driver. He denied any contact with a patient with PTB.

On examination, he was spiking high-grade fever with a temperature of $39.2^{\circ} \mathrm{C}$; tachypneic with a respiratory rate of 30 and tachycardic with a heart rate ranging between 120-130. His blood pressure was within normal limits. The patient was cachectic. Neck examination was unremarkable. Chest examinations showed decreased air entry in the left side in all lung zone with coarse crackles and dull percussion notes. The rest of the physical examination was unremarkable.

His laboratory findings on admission showed mild leukocytosis and anemia with white blood cells (WBCs) level of $11.8 \times 10^{\wedge} 3 / \mathrm{uL}$ and $\mathrm{Hb}$ level of $11.4 \mathrm{mg} / \mathrm{dL}$. platelet count with normal range. Other labs showed 


\section{Cureus}

moderate hyponatremia with sodium level $124 \mathrm{mmol} / \mathrm{L}$ with CRP of high with value $140 \mathrm{mg} / \mathrm{L}$. Renal and liver function tests were unremarkable.

Chest radiography showed evidence of heterogeneous opacity involving the whole left lung suggestive of consolidation and evidence of a cavitary lesion in the left upper zone (Figure 1).

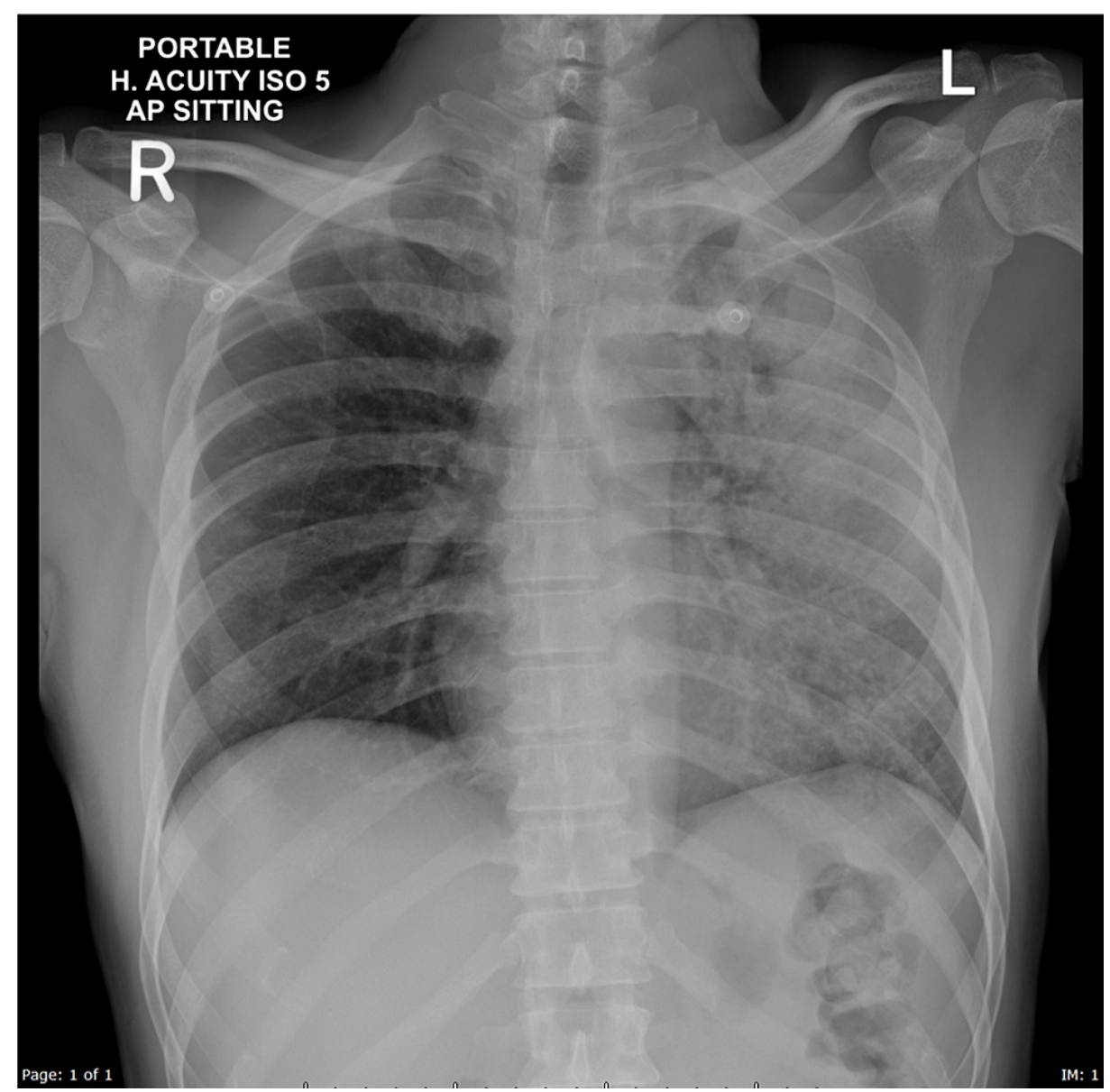

FIGURE 1: Frontal radiograph of the chest, demonstrating diffuse left lung field consolidation with an air bronchogram and evidence of a cavitary lesion in the left upper zone

His sputum smear for acid-fast bacilli (AFB) and polymerase chain reaction (PCR) was positive for

Mycobacterium tuberculosis and was started on anti-tuberculous therapy (ATT). His HIV test was negative.

After 24 hours, the patient was still complaining of shortness of breath. Further assessment showed that he still had tachycardia. The further blood test showed raised troponin-T and pro-B-type natriuretic peptide (pro-BNP) with values of $24 \mathrm{ng} / \mathrm{L}$ and $5,634 \mathrm{pg} / \mathrm{mL}$, respectively. The thyroid function test was normal. His electrocardiogram (ECG) showed sinus tachycardia with S1Q3T3 (Figure 2). 


\section{Cureus}

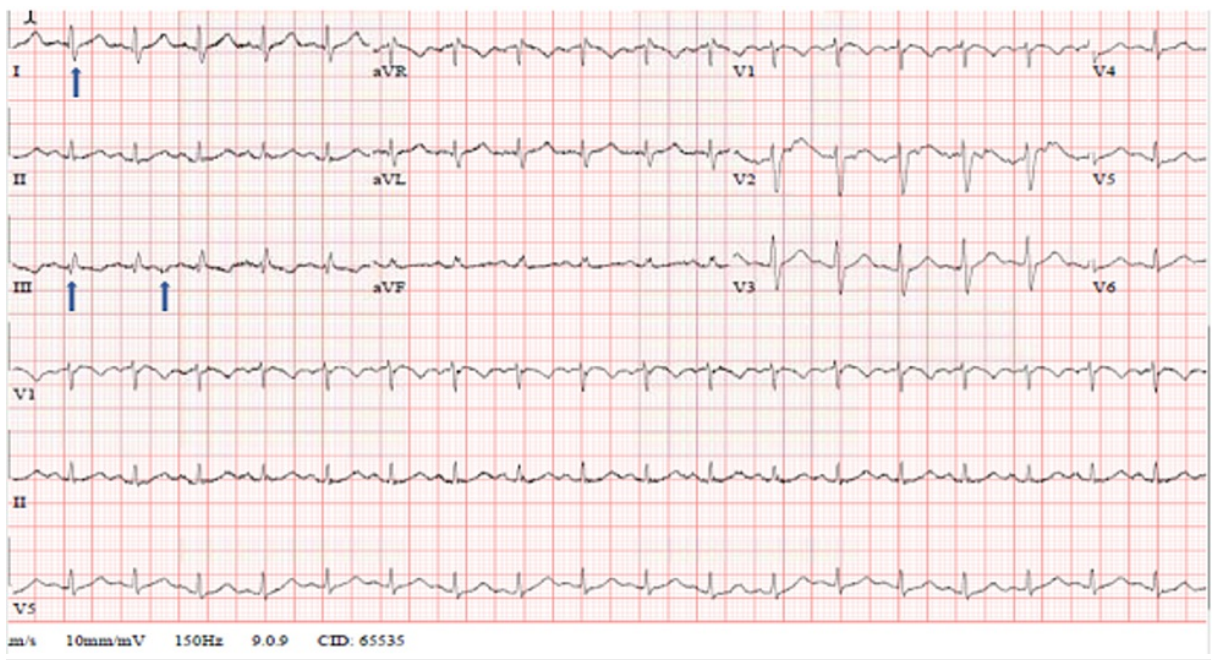

FIGURE 2: ECG strip showing the pattern of S1Q3T3

ECG: electrocardiogram

Repeated physical examination revealed new mild right leg swelling, which was not present on the initial assessment. His Doppler ultrasound showed evidence of acute DVT from the distal posterior tibial vein (PTV) up to proximal superficial femoral vein (SFV) with areas of partial and no flow (Figure 3).
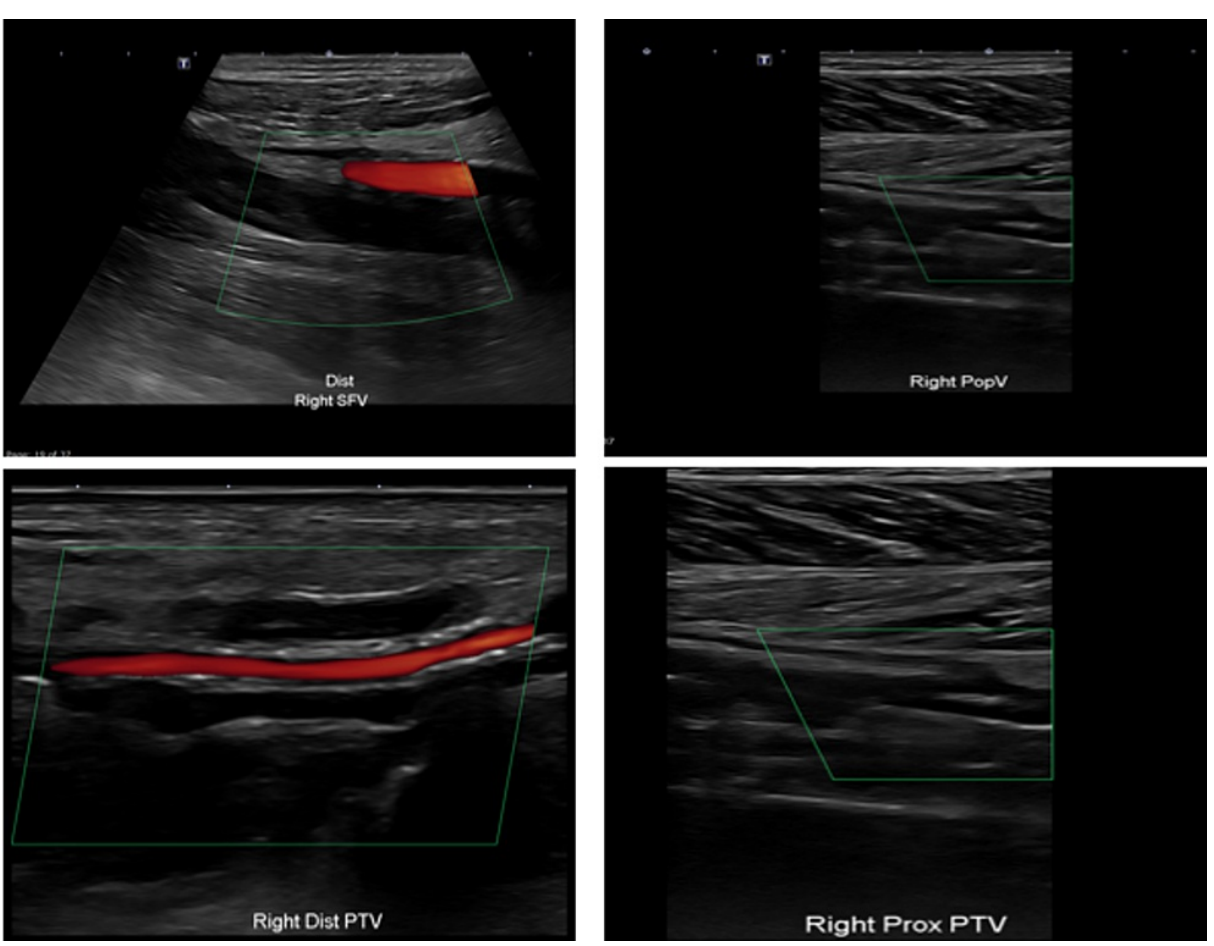

FIGURE 3: Right lower limb Doppler US scan showing no flow within the SFV, popliteal vein, proximal and distal PTV, compatible with right lower limb DVT

SFV: superficial femoral vein; posterior tibial vein: PTV; DVT: deep vein thrombosis

Chest CT pulmonary angiogram (CTPA) showed saddle embolism extending to both pulmonary arteries and their major divisions (Figure 4). Echocardiogram showed normal global systolic LV function (ejection fraction (EF) 52\%), moderate-severely dilated right ventricle (RV), and severely reduced RV function associated with septal motion suggestive of RV pressure overload. His right atrium is moderately dilated, and the pulmonary artery pressure is severely increased. The patient was moved to a high dependency unit 


\section{Cureus}

and was given thrombolysis therapy (alteplase) as per the local protocol. He showed significant improvement in his clinical condition with improvement in his heart rate and shortness of breath.
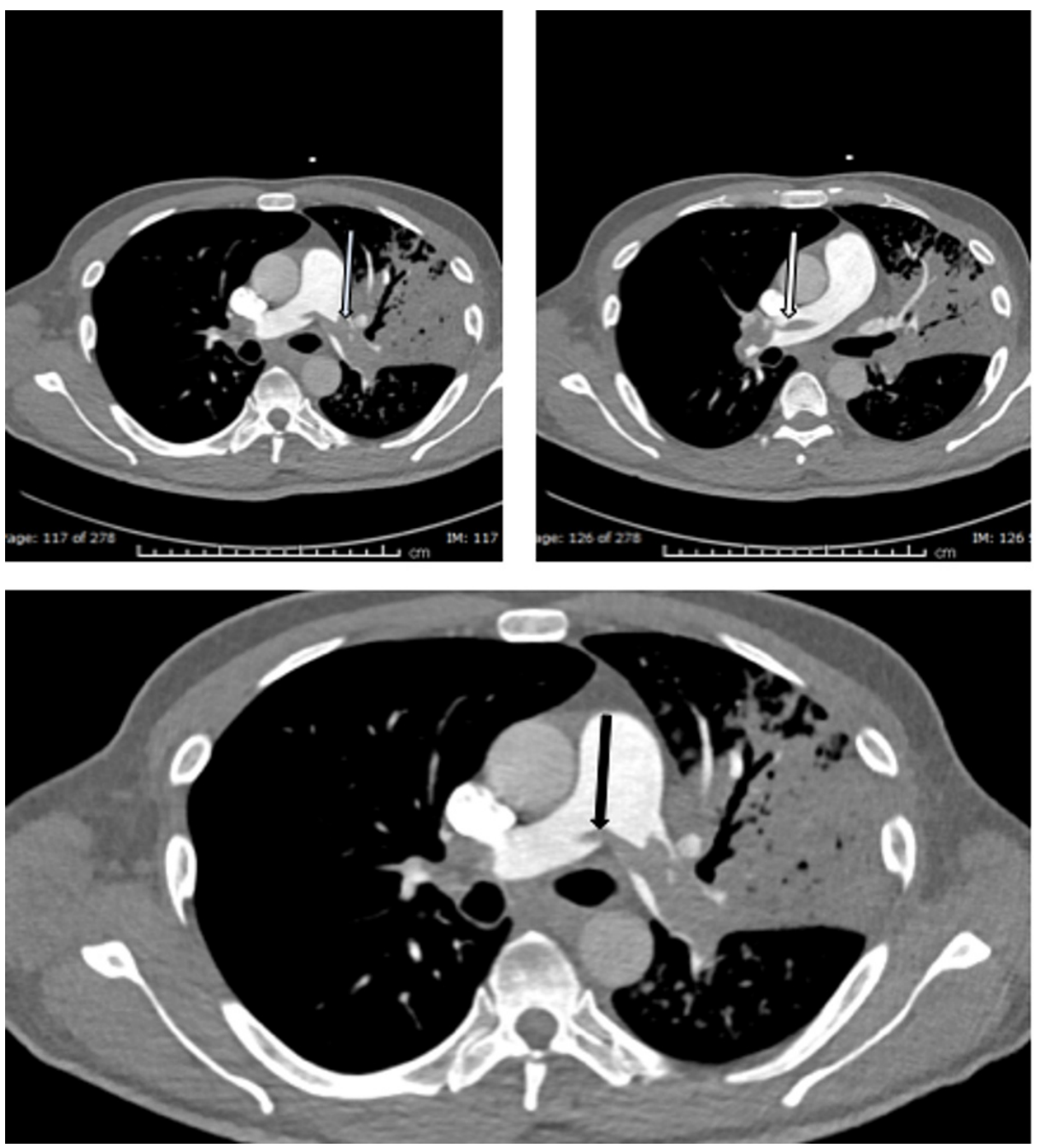

FIGURE 4: CT pulmonary angiogram (PA) demonstrating large filling defect within the main pulmonary artery (black arrow), which is extending to the right (white arrow) and left (blue arrow) pulmonary arteries representing saddle pulmonary embolism

He was started with anticoagulation as per the local protocol with enoxaparin and warfarin. The plan is to follow the local isolation protocol for PTB and optimize his international normalized ratio (INR) within the therapeutic range.

\section{Discussion}

Our case is a clear example of pulmonary tuberculosis, which was complicated by unstable pulmonary embolism (PE) and deep vein thrombosis (DVT). He was treated with thrombolysis successfully. VTE can occur at the time of presentation of PTB or later in the course of the disease. Although VTE is very rare, the thrombogenic potential of tuberculosis has been reported in the literature before [3-4,6]. Other sites where VTE can occur in patients with TB are the hepatic veins [7] and cerebral venous sinuses [8].

Venous thromboembolism in patients with tuberculosis can be due to different causes. One of the most common causes is an inflammatory reaction that triggers elevated fibrinogen levels with impaired fibrinolysis, which is coupled with a decrease in the antithrombin III level and reactive thrombocytosis [910]. Secondly, patients with severe tuberculosis are often dehydrated. According to Poiseuille's equation, when fluid in plasma is diminished due to dehydration, blood viscosity will increase and result in a reduced blood flow and increased prothrombotic state $[9,11]$. The third reason is attributed to the use of antitubercular drugs such as rifampicin. This drug is associated with a four times higher risk of deep vein thrombosis in a retrospective analysis of 1366 adult TB patients [9,12]. The occurrence of thrombosis usually 
happened two weeks from the commencement of antitubercular drugs [9]. Lastly, enlarged lymph nodes due to cell-mediated immunity can cause compressive effects on blood vessels, which result in vascular thrombosis [9,13-14].

Our case showed an unstable PE in a patient with a very clear diagnosis of PTB, which can be easily missed due to the nature of symptoms that are common to both conditions; however, unexplained tachycardia, mild leg swelling on careful examination, with raised a troponin level helped us investigate for VTE. Actually, even though there are many risk factors for PE, it is easy to miss it due to the poor specificity of the symptoms [15]. In acute PE, elevated heart rate is not only a diagnostic marker but also serves as one of the prognostic factors for mortality outcome, principally in-hospital mortality [16-17].

The patient was thrombolysed successfully with alteplase $(50 \mathrm{mg}$ ) as per local protocol due to unstable PE as indicated by CTPA and echocardiogram findings. He tolerated it very well. Therefore, thrombolysis with alteplase should be considered in such patients with a low risk of bleeding or no contraindications. Another challenge in these patients is the drug interaction between ATT and anticoagulants like warfarin due to the effects of rifampicin on drug metabolism through cytochrome P450 [18]. Our patient was started on a therapeutic dose of enoxaparin (LMWH) and warfarin until the targeted INR level was achieved along with ATT. He will be referred to an anticoagulation clinic and infectious disease clinic after discharge.

\section{Conclusions}

This case report highlights the increased risk of VTE in patients with pulmonary tuberculosis due to a hypercoagulable state associated with tuberculosis. This can lead to increased mortality, which is potentially preventable by prompt diagnosis and treatment. It also highlights the fact that thrombolysis is welltolerated in patients with unstable PE with PTB who have no contraindications.

\section{Appendices \\ Data availability statement}

The data that support the findings of this study are available from the corresponding author upon reasonable request.

\section{Additional Information \\ Disclosures}

Human subjects: Consent was obtained or waived by all participants in this study. Conflicts of interest: In compliance with the ICMJE uniform disclosure form, all authors declare the following: Payment/services info: All authors have declared that no financial support was received from any organization for the submitted work. Financial relationships: All authors have declared that they have no financial relationships at present or within the previous three years with any organizations that might have an interest in the submitted work. Other relationships: All authors have declared that there are no other relationships or activities that could appear to have influenced the submitted work.

\section{Acknowledgements}

The authors would like to acknowledge the Internal Medicine Residency program at Hamad Medical Corporation for its support. They would also like to acknowledge the following author contributions: Dr. Eihab A. Subahi and Ijaz Kamal wrote and edited the manuscript. Dr. Mouhammad Jassem Alawad and Dr. Elabbass A. Abdelmahmuod were responsible for the literature review. Dr. Dalal Sibira provided us with labeled radiological images.

\section{References}

1. Ha H, Kim KH, Park JH, et al.: Thromboembolism in Mycobacterium tuberculosis Infection: analysis and Literature Review. Infect Chemother. 2019, 51:142-9. 10.3947/ic.2019.51.2.142

2. Ben Amar J, Dahri B, Aouina H, Bouacha H: Venous thromboembolism in patients with acute tuberculosis [Article in French]. Rev Pneumol Clin. 2015, 71:327-34. 10.1016/j.pneumo.2015.04.004

3. Goncalves IM, Alves DC, Carvalho A, Brito MDC, Calvario F, Duarte R: Tuberculosis and venous thromboembolism: a case series. Cases J. 2009, 16:1-4.

4. Suárez Ortega S, Artiles Vizcaíno J, Balda Aguirre I, Melado Sánchez P, Arkuch Saade ME, Galán AE, Betancor León P: Tuberculosis as risk factor for venous thrombosis. An Med Interna. 1993, 10:398-400

5. Lee CR, Thrasher KA: Difficulties in anticoagulation management during coadministration of warfarin and rifampin. Pharmacotherapy. 2001, 21:1240-6. 10.1592/phco.21.15.1240.33897

6. Naithani R, Agrawal N, Choudhary VP: Deep venous thrombosis associated with tuberculosis . Blood Coagul Fibrinolysis. 2007, 18:377-80. 10.1097/MBC.0b013e3280d942b4

7. Grover SB, Gogna A, Grover SB, Arun S, Saluja S: Isolated hepatic inferior vena cava thrombosis in a case of tuberculosis - case report. JIACM. 2004, 5:266-8.

8. Fiorot Júnior JA, Felício AC, Fukujima MM, Rodrigues CA, Morelli VM, Lourenço DM, Prado GF: Tuberculosis: an uncommon cause of cerebral venous thrombosis? . Arq Neuropsiquiatr. 2005, 63:852-4. 


\section{Cureus}

$10.1590 / \mathrm{s} 0004-282 \times 2005000500025$

9. Huei TJ, Henry TCL, Ho CA, Mohamad Y: A rare case of ileocecal tuberculosis with pulmonary embolism and deep vein thrombosis. J Clin Diagn Res. 2017, 11:PD03-4. 10.7860/JCDR/2017/27923.10192

10. Robson SC, White NW, Aronson I, Woollgar R, Goodman H, Jacobs P: Acute-phase response and the hypercoagulable state in pulmonary tuberculosis. Br J Haematol. 1996, 93:943-9. 10.1046/j.13652141.1996.d01-1722.x

11. Singh S, Randle LV, Callaghan PT, Watson CJ, Callaghan CJ: Beyond Poiseuille: preservation fluid flow in an experimental model. J Transplant. 2013, 2013:605326. 10.1155/2013/605326

12. White NW: Venous thrombosis and rifampicin. Lancet. 198919, 334:434-5. 10.1016/S0140-6736(89)90603-X

13. Gogna A, Pradhan GR, Sinha RS, Gupta B: Tuberculosis presenting as deep vein thrombosis. Postgrad Med J. 1999, 75:104-5. 10.1136/pgmj.75.880.104

14. Sharma A, Sharma V: Abdominal aortic thrombosis and tuberculosis: an uncommon association . Gastroenterol Rep (Oxf). 2014, 2:311-2. 10.1093/gastro/gou021

15. Howard LS, Barden S, Condliffe R, et al.: British Thoracic Society Guideline for the initial outpatient management of pulmonary embolism. BMJ Open Respir Res. 2018, 5:e000281. 10.1136/bmjresp-2018000281

16. Ahmedy F, Ahmad Fauzi A, Engkasan JP: Asymptomatic tachycardia and acute pulmonary embolism in a case of tuberculosis spondylodiscitis. Spinal Cord Ser Cases. 2018, 4:43. 10.1038/s41394-018-0074-7

17. Keller K, Beule J, Coldewey M, Dippold W, Balzer JO: Heart rate in pulmonary embolism. Intern Emerg Med. 2015, 10:663-9. 10.1007/s11739-015-1198-4

18. Sekaggya C, Nalwanga D, Von Braun A, et al.: Challenges in achieving a target international normalized ratio for deep vein thrombosis among HIV-infected patients with tuberculosis: a case series. BMC Hematol. 2016, 16:16. 10.1186/s12878-016-0056-6 